Revista de Iniciação Científica em Odontologia. 2018;16(6):13-20

ISSN 1677-3527 - DOI: 10.4034/Revico.2018.16.6.2

\title{
Pesquisa
}

\section{Análise da produção e satisfação dos usuários nos CEO da Paraíba}

\author{
Analysis of the production and user satisfaction in the CEOs of Paraíba
}

Amanda de Oliveira Pereira ${ }^{1}$; Thiago Lucas da Silva Pereira ${ }^{1}$; Wilton Wilney Nascimento Padilha ${ }^{3}$.

${ }^{1}$ Acadêmico de Odontologia Universidade Federal da Paraíba, João Pessoa-Paraíba-Brasil. 2Professor do Programa de Pós-Graduação em Odontologia da UFPB, João Pessoa, PB, Brasil.

Autor e endereço para correspondência:

Wilton Wilney Nascimento Padilha;Rua Miguel Satyro, 350, Cabo Branco, João PessoaCEP:58045110,Wilton.padilha@yahoo.com.br.

\section{Resumo}

Introdução: Dados de monitoramento de produção e de satisfação do usuário são meios de se conhecer o nível de atendimento das necessidades de saúde bucal. Objetivo: Conhecer o perfil da produção especializada em odontologia na Paraíba e verificar a relação com a satisfação dos usuários. Metodologia: Estudo transversal de abordagem indutiva, procedimento estatístico-comparativo e técnica de documentação indireta. No Sistema de Informação Ambulatorial (SIA/SUS), identificou-se a produção dos Centros de Especialidades Odontológicas (CEO) da Paraíba ( $N=78$ ) em 2016. Foram retirados da plataforma DABSAUDE, os dados referentes à terceira fase do PMAQ-CEO $(N=53)$, analisando a satisfação do usuário $(n=530)$. Foram excluídos os CEO não cadastrados no PMAQ-CEO $(\mathrm{N}=25)$. A produção foi analisada de acordo com a Portaria 1.464 do Ministério da Saúde, que regulamenta a quantidade e o tipo de procedimentos realizados. Foi analisada a satisfação de 10 usuários por CEO a partir das médias das notas das avaliações, que iam de 1 (muito bom) a 5 (muito ruim). A análise foi descritiva e analítica, utilizando o teste de correlação de Spearman com as variáveis de produção total e nível de satisfação, foram descritos a produção por especialidade e por tipo de CEO. Resultados: O cumprimento de metas é mais efetivo nos CEO tipo 3 nas especialidades de cirurgia oral e procedimentos em PNE, no tipo 2 em periodontia e no tipo 1 em endodontia. Conclusão: Não há correlação entre o número de procedimentos realizados e a satisfação do usuário. O número de procedimentos realizados nos três tipos de CEO não atinge a meta proposta.

Descritores: Atenção Secundária à Saúde. Sistema Único de Saúde. Acesso aos serviços de saúde. 


\begin{abstract}
Introduction: Productivity monitoring and user satisfaction data are ways to know the supply level of the oral health demandings. Objective: Meet the profile of the productivity in dental specialties in Paraíba and verify the relationship between the offered services and the satisfaction of users. Methodology: Cross-sectional study with inductive approach, statistical-comparative procedure and indirect documentation technique. The productivity of the Paraíba Dental Specialties Centers (CEO) $(N=78)$ was obtained in 2016 by the Outpatient Information System (SIA / SUS). Data from the third phase of PMAQ-CEO $(\mathrm{N}=53)$ were extracted from the DABSAUDE platform to analyze the user satisfaction $(n=530)$. Non-registered on PMAQ-CEO CEOs $(N=25)$ were excluded. The productivity was analyzed according to the decree 1,464 of the Brazil Ministry of Health, which regulates quantity and type of performed procedures. The satisfaction of 10 users per CEO was analyzed from the averages of the evaluation scores, which ranged from 1 (very good) to 5 (very poor). The analysis was descriptive and analytical, using the Spearman correlation test with variables of total productivity and level of user satisfaction; the productivity was described by the CEO's type and offered specialties on it. Results: Type 3 CEOs have a more effective goal compliance in minor oral surgery and specialneeding patients procedures; Type 2, in periodontics; type 1, in endodontics. Conclusion: There isn't a correlation between the quantity of procedures performed and user satisfaction. The number of procedures performed in the three types of CEOs does not reach the recommended goal.
\end{abstract}

Key-words: Secondary Care. Unified Health System. Health Services Accessibility.

\title{
Introdução
}

A Política Nacional de Saúde Bucal - Brasil Sorridente institui a expansão da atenção básica, com o aumento do nível de complexidade dos procedimentos oferecidos e o cuidado em atenção especializada aos usuários. A proposta da política é reorientar o modelo de odontologia mutiladora para uma odontologia preventiva, centrada na atenção básica como chave para promoção e prevenção em saúde. Dessa forma, se faz necessário que o incremento no cuidado básico seja acompanhado pelo aumento de produção em cuidados secundários, possibilitando a otimização do sistema de referências e contra-referencias ${ }^{1,2}$.

A integração entre atenção primária, secundária e terciária garante ao usuário a integralidade do cuidado na saúde bucal. A atenção básica permanece como a principal integrante do sistema, onde deve haver acolhimento, atendimento das demandas cabíveis e o correto encaminhamento para outros níveis de saúde em procedimentos complexos. A atenção secundária em saúde bucal é fornecida nos Centros de Especialidades Odontológicas (CEO), onde são 
preconizados o atendimento em periodontia, endodontia, cirurgia oral, diagnóstico bucal e atendimento básico a portadores de necessidades especiais ${ }^{1}$.

Os CEO estão inclusos no Cadastro Nacional de Estabelecimentos de Saúde (CNES) e devem cumprir metas de produção em cada especialidade, sendo estas reguladas pela Portaria $n^{\circ}$ 1.464, de junho de 2011. O não cumprimento das metas estabelecidas por dois meses consecutivos ou três meses intercalados no período de um ano pode ocasionar a suspensão de recursos para o CEO. Os registros no CNES são de domínio público e podem ser acessados no portal DATASUS².

Considerando o monitoramento adequado dos recursos destinados à saúde, bem como da satisfação dos profissionais e usuários da rede, se estabeleceu o Programa Nacional de Melhoria do Acesso e da Qualidade dos Centros de Especialidades Odontológicas (PMAQ-CEO). O programa é dividido em quatro fases: adesão e contratualização; desenvolvimento; avaliação externa e re-contratualização. Os CEO que aderem ao programa passam a receber $20 \%$ de incentivo financeiro, após a avaliação externa, ele pode perder o incentivo, manter ou ampliar entre $60 \%$ e $100 \%$.

A análise da satisfação dos usuários e as avaliações de produção são ferramentas uteis no processo de desenvolvimento e aprimoramento dos serviços da atenção secundária. A perspectiva do usuário é significativamente utilizada, mostrando a realidade dos centros de especialidades e do padrão de serviço oferecido ao usuário, auxiliando gestores nas etapas de detecção dos problemas $^{3}$. A atual configuração da regionalização da saúde na Paraíba organiza o estado em 16 regiões de saúde distribuídas em 04 Macrorregiões, contemplando os seus 223 municípios. O estado tem 88 Centros de Especialidades Odontológicas em funcionamento atualmente, abrangendo todas as suas regiões de Saúde.

O cenário analisado dos CEO da Paraíba é em geral insatisfatório em se tratando dos cumprimentos de metas estabelecidos na portaria. Há grande disparidade entre a quantidade de núcleos de atenção básica e de Centros de Especialidades Odontológicas, gerando uma alta demanda reprimida. Além do baixo registro de procedimentos, corre ainda problemas na notificação dos procedimentos, havendo registros que deveriam ser da atenção básica e outros de alta complexidade que seriam gerados em nível hospitalar, e não ambulatorial ${ }^{4}$.

O objetivo do estudo é o reconhecimento de aspectos referentes à produção de procedimentos nos CEO da Paraíba e dados da terceira fase do PMAQ-CEO, usando a satisfação do usuário como instrumento de análise. 


\section{Metodologia}

Estudo transversal de abordagem indutiva, procedimento estatístico-comparativo e técnica de documentação indireta. Os dados de produção em procedimentos dos CEO foram retirados da plataforma TABWIN, disponível no site DATASUS, referentes ao ano de 2016. O universo foi composto pelos 78 CEO do estado e a amostra foi de 53 CEO, sendo excluídos aqueles que não aderiram ao PMAQ-CEO. Os CEO foram separados de acordo com o tipo, uma vez que essa separação permite identificar o recurso financeiro que este CEO recebe (Quadro 1).

\begin{tabular}{|c|c|c|c|}
\hline \multicolumn{4}{|c|}{ Quadro 1. Recursos destinados aos CEO de acordo com o tipo. } \\
\hline & $\begin{array}{l}\text { Número de cadeiras } \\
\text { odontológicas }\end{array}$ & $\begin{array}{l}\text { Incentivo de implantação do } \\
\text { CEO }\end{array}$ & $\begin{array}{l}\text { Incentivo de custeio } \\
\text { mensal }\end{array}$ \\
\hline CEO tipo 1 & 3 & $\mathrm{R} \$ 60.000$ & $\mathrm{R} \$ 8.250$ \\
\hline CEO tipo 2 & $4-6$ & $\mathrm{R} \$ 75.000$ & $\mathrm{R} \$ 11.000$ \\
\hline CEO tipo 3 & $>7$ & $R \$ 120.000$ & $\mathrm{R} \$ 19.250$ \\
\hline
\end{tabular}

A análise foi feita por tipo de CEO de acordo com a produção de metas estabelecidas pela portaria 1464/GM, de 24 de junho de 2011 (Quadro 2).

\begin{tabular}{|c|c|c|c|}
\hline \multicolumn{4}{|c|}{ Quadro 2. Metas de produção mensal nas diversas especialidades de acordo com o tipo de CEO. } \\
\hline & CEO tipo 1 & CEO tipo 2 & CEO tipo 3 \\
\hline Procedimentos em periodontia/ mês & 60 & 90 & 150 \\
\hline Procedimentos em endodontia/ mês & 35 & 60 & 95 \\
\hline $\begin{array}{c}\text { Procedimentos básicos realizados em pessoas } \\
\text { com necessidades especiais/ mês }\end{array}$ & 80 & 110 & 190 \\
\hline Procedimentos em Cirurgia oral/ mês & 80 & 90 & 170 \\
\hline $\begin{array}{c}\text { Procedimentos em endodontia em dentes com } \\
\text { três raízes ou mais/ mês }\end{array}$ & 7 & 12 & 19 \\
\hline $\begin{array}{c}\text { Procedimentos restauradores realizados em } \\
\text { pessoas com necessidades especiais/ mês }\end{array}$ & 40 & 55 & 95 \\
\hline
\end{tabular}

Foi analisada a satisfação de 10 usuários de cada CEO a partir das médias das notas das avaliações, que iam de 1 (muito bom) a 5 (muito ruim). Os dados de satisfação foram extraídos do segundo ciclo do PMAQ-CEO. Foi realizado teste de correlação de Spearman para avaliar produção total dos CEO e satisfação do usuário. Foi excluído do teste um CEO por este ter apresentado produção zerada. 


\section{Resultados}

O cumprimento de metas nos três tipos de CEO se mostrou aquém do estipulado pela portaria de regulamentação (Tabela 1). Os centros com melhor resultado são do tipo 3 , apesar do seu desempenho em endodontia ser o menor. É possível detectar uma clara deficiência na especialidade de endodontia, sendo sua meta cumprida em 2 meses do ano.

\section{Tabela 1. Média do cumprimento de meta, em meses, no ano de 2016 por tipo de CEO da Paraíba.}

$\begin{array}{ccccc} & \begin{array}{c}\text { Procedimentos } \\ \text { básicos em PNE }\end{array} & \text { Cirurgia oral } & \text { Endodontia } & \text { Periodontia } \\ \text { CEO tipo 1 } & 5 & 2,9 & 2,4 & 5,1 \\ \text { CEO tipo 2 } & 5,1 & 4 & 1,3 & 4,7 \\ \text { CEO tipo 3 } & 9,5 & 6 & 1 & 4 \\ \text { Total } & 5,4 & 3,4 & 2 & 5\end{array}$

Os dados de produção absoluta apresentam um déficit em relação ao que deveria ser feito nos CEO tipo 1, onde se cumpre $83,2 \%$ do ideal e tipo 2, com $76,4 \%$. Os CEO tipo 3 apresentam produção absoluta 76\% maior que o mínimo estipulado, no entanto, o alto desvio-padrão aponta para uma desigualdade significativa na distribuição desses procedimentos.

\begin{tabular}{ccccc}
\hline \multicolumn{5}{c}{ Tabela 2. Dados de produção anual dos CEO da Paraíba em 2016. } \\
\hline & $\begin{array}{c}\text { Número de CEO da } \\
\text { amostra }\end{array}$ & $\begin{array}{c}\text { Produção absoluta } \\
\text { de procedimentos }\end{array}$ & $\begin{array}{c}\text { Média de procedimentos } \\
\text { por CEO }\end{array}$ & Desvio-padrão \\
CEO tipo 1 & 36 & 91.665 & 2546,2 & 1501,8 \\
CEO tipo 2 & 13 & 41.715 & 3208,8 & 2078,5 \\
CEO tipo 3 & 4 & 67.074 & 16768,5 & 14196,7 \\
Total & 53 & 200.454 & 3782,1 & 5315,5
\end{tabular}

A satisfação do usuário do usuário ficou na média entre muito bom e bom (Tabela 5), tendo fraca correlação com a produção total de acordo com o teste de correlação de Spearman.

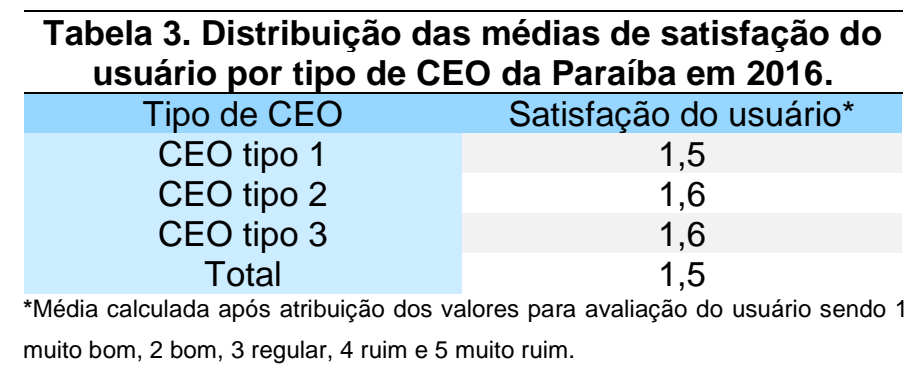




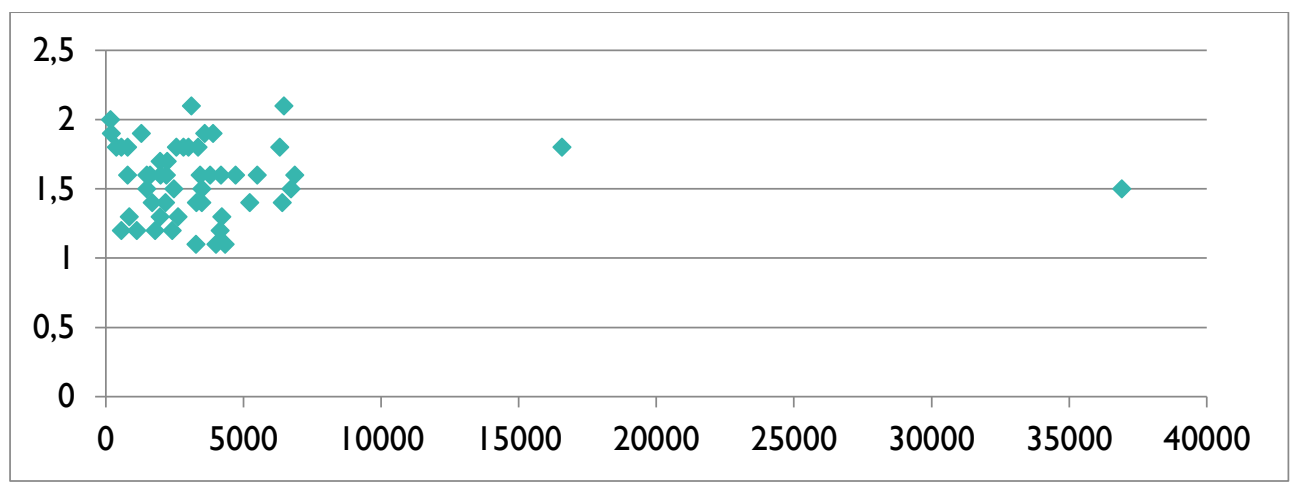

Figura 1. Correlação entre o nível de satisfação dos usuários dos CEO da Paraíba em 2016 e a produção absoluta de procedimentos.

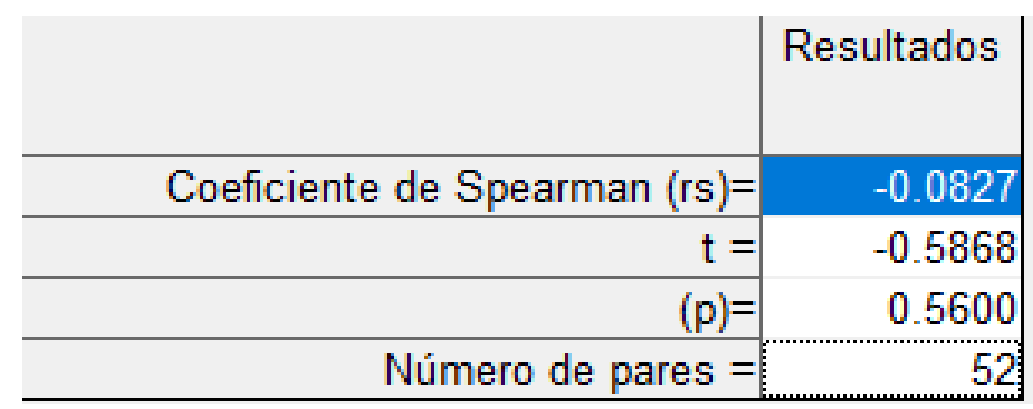

Figura 2. Teste de correlação de Spearman sobre satisfação do usuário e número de procedimentos.

\section{Discussão}

A implementação dos Centros de Especialidades Odontológicas é uma forma de complementar a assistência fornecida pela atenção básica. A relação entre esses níveis tem como intuito oferecer a integralidade do tratamento do usuário. O sistema funciona de maneira ideal quando a atenção básica recepciona o indivíduo e o referência para o CEO quando necessário, recebendo-o de volta quando o tratamento especializado é concluído, realizando a prevenção de novas enfermidades. Assim, o funcionamento adequado de todos os níveis é importante para o andamento do sistema como um todo ${ }^{5}$.

Entre as justificativas para a pouca oferta do serviço de endodontia (Tabela 1) se encontram o baixo número de equipamentos e profissionais habilitados, bem como a necessidade de várias sessões para a conclusão de um tratamento endodôntico. Outro fator que interfere negativamente na especialidade é que no momento em que o canal é tratado e a dor cessa, muitos pacientes 
abandonam o tratamento mesmo sem estar concluído, impedindo seu registro no sistema ${ }^{5}$. Uma saída plausível para aperfeiçoar o atendimento na especialidade seria a implementação de instrumentos rotatórios de NiTi nos CEO, que possibilitam a conclusão do tratamento em menos tempo e, consequentemente, permitem que mais pessoas sejam atendidas ${ }^{6}$.

De acordo com a SB Brasil 2010 a prevalência de pessoas com doença periodontal moderada a grave no país é de 15,3\%, mesmo assim, CEO não realizam a meta estipulada em periodontia (Tabela 1). Entre os desafios para realização dos procedimentos periodontais, além das condições estruturais, há a grande necessidade da colaboração do paciente para que estes possam ser realizados. Entre as metas para a periodontia, apenas a raspagem corono-radicular é uma técnica não-cirúrgica, portanto, todas as outras (gengivectomia, gengivoplastia, procedimento cirúrgico periodontal e enxerto gengival) requerem do paciente uma boa condição periodontal prévia, que muitas vezes não são obtidas graças a fatores extrínsecos ao cuidado do dentista, como hábitos de higiene ruins, tabagismo e etilismo7.

Os procedimentos básicos em pacientes com necessidades especiais são, em sua maioria, focados na prevenção. No entanto, fatores ligados à dificuldade de acesso ao serviço odontológico fazem com que muitos desses pacientes cheguem ao serviço com diversos problemas instalados. Existem inúmeros fatores que predispõem essas pessoas a problemas bucais, como a dificuldade de higienização, respiração bucal, má oclusão e uso crônico de medicamentos ${ }^{8}$. Uma vez inseridos no serviço, esses usuários contam ainda com problemas como a falta de estrutura adaptada às suas necessidades e carência de profissionais que saibam lidar com esses pacientes. É necessário que haja maior incentivo à capacitação e especialização na área, além da adaptação dos CEO para a circulação de deficientes físicos ${ }^{8}$.

Uma das dificuldades encontradas na análise dos dados referentes à produção é o uso de dados secundários. Não é incomum que o registro seja feito de maneira errada, havendo procedimentos básicos e de alta complexidade sendo notificados em centros de especialidades ${ }^{9}$. Esse viés é importante para a percepção de que nem todos os CEO que realizam a notificação de diversos procedimentos se enquadram nas normas preconizadas pelo Ministério da saúde.

A ausência de correlação ao teste de Spearman se justifica a partir da média de satisfação em todos os CEO ser alta. Levando-se em consideração que satisfação do usuário que ficam entre satisfeitos e muito satisfeitos (Tabela 3) é possível haver um o viés de gratidão. Por haver uma maioria de usuários pertencentes a classes sociais sem acesso a outros serviços para utilizar como referência de qualidade, há tendência em manifestar satisfação mesmo quando não recebem um 
tratamento ideal. Dessa forma, é importante mensurar a satisfação do usuário, mas esta deve ser avaliada em conjunto com outros dados na avaliação da qualidade do $\mathrm{CEO}^{10}$.

\section{Conclusão}

Os três tipos de CEO não atingem as metas de produção estipuladas pela portaria de regulamentação. Ao CEO tipo um são os melhores no cumprimento das metas em endodontia e periodontia. Os CEO tipo três são os melhores em cirurgia oral e procedimentos básicos em pacientes com necessidades especiais. A satisfação do usuário não foi um bom parâmetro para avaliar a qualidade dos CEO, uma vez que mesmo aqueles com baixa produção obtiveram notas altas de satisfação.

\section{Referências}

1. Ministério da Saúde. Portaria n‥599. Define a implantação de especialidades odontológicas (CEO) e de laboratórios regionais de próteses dentárias (LRPDs) e estabelece critérios, normas e requisitos para seu credenciamento. Diário Oficial da União 2006; 24 mar

2. Kitamura ES, Bastos RR, Palma PV, Leite ISG. Avaliação da satisfação dos usuários dos Centros de Especialidades Odontológicas da macrorregião Sudeste de Minas Gerais, 2013. Epidemiol. Serv. Saúde. 2016; 25(1):137-148.

3. Esperidião MA, Trad LAB. Avaliação de satisfação de usuários: considerações teóricoconceituais. Cad. Saúde Pública. 2006; 22(6):1267-1276.

4. Freitas CHSM, Lemos GA, Pessoa TRRF, Araujo MF, Forte FDS. Atenção em saúde bucal: avaliação dos centros de especialidades odontológicas da Paraíba. Saúde debate. 2016; 40(108):131-143.

5. Martins RC, Seijo MOS, Ferreira EF, Paiva SM, Ribeiro Sobrinho AP. (2012). Dental students' perceptions about the endodontic treatments performed using NiTi rotary instruments and hand stainless steel files. Brazilian Dental Journal. 2012; 23(6):729-736.

6. Vettore MV, Marques RAA, Peres MA. Desigualdades sociais e doença periodontal no estudo SBBrasil 2010: abordagem multinível. Rev. Saúde Pública. 2013; 47(Suppl 3):29-39.

7. Domingues NB, Ayres KCM, Mariusso MR, Zuanon ACC, GIRO EMA. Caracterização dos pacientes e procedimentos executados no serviço de atendimento a pacientes com necessidades especiais da Faculdade de Odontologia de Araraquara - UNESP. Rev. odontol. UNESP. 2015; 44(6): 345-350.

8. Thomaz EBAF, Sousa GMC, Queiroz RCS, Coimbra LC. Avaliação do cumprimento das metas de produtividade em Centros de Especialidades Odontológicas no Maranhão, 2011. Epidemiol. Serv. Saúde. 2016; 25(4):807-818.

9. Machado FCA, Silva JV, Ferreira MAF. Fatores relacionados ao desempenho de Centros de Especialidades Odontológicas. Ciênc. saúde coletiva. 2015; 20(4):1149-1163.

10. Vaitsman J, Andrade GRB. Satisfação e responsividade: formas de medir a qualidade e a humanização da assistência à saúde. Ciênc. saúde coletiva. 2005; 10(3):599-613. 